\title{
The next 10 years: Challenges for the future and overcoming resistance to targeted therapies for renal cell carcinoma
}

\author{
Daniel Y.C. Heng, MD, FRCPC \\ Tom Baker Cancer Centre, Calgary, and Department of Medicine, University of Calgary, Calgary, AB, Canada
}

Cite as: Can Urol Assoc J 2016;10(11-12Supp17):S256-8.

http://dx.doi.org/10.5489/cuaj.4294

\section{Abstract}

The introduction of targeted therapies over the past 10 years revolutionized the treatment of metastatic renal cell carcinoma (mRCC). The next 10 years hold promise for even greater expansion of the therapeutic armamentarium for mRCC. A number of recently completed and ongoing trials have explored the use of antivascular endothelial growth factor receptor (VEGFR) tyrosine kinase inhibitors (TKIs) and mammalian target of rapamycin (mTOR) inhibitors in the adjuvant setting, the use of predictive biomarkers to guide personalized medicine, as well as new systemic treatments and combination therapies for mRCC.

\section{Introduction}

Over the past decade, the treatment of metastatic renal cell carcinoma (mRCC) has evolved to include drugs that target the vascular endothelial growth factor (VEGF) and mammalian target of rapamycin (mTOR) pathways, resulting in substantially improved outcomes for this patient population. However, despite prolonged survival, the majority of patients will develop resistance to VEGF- and mTOR-targeted therapies. New therapeutic strategies are continually being explored to overcome resistance and provide a more robust response.

New paradigms in the treatment of RCC are developing. Of particular interest are the use of targeted therapies in the adjuvant setting and the use of biomarkers to improve patient selection and guide personalized medicine. The next 10 years will also likely see the introduction of additional treatments for mRCC, including new systemic treatments and new combination therapies.

\section{Use of targeted therapies in the adjuvant setting}

There is currently no defined role for adjuvant therapy for localized RCC following partial or radical nephrectomy.
Several adjuvant strategies, including cytokine therapy, radiotherapy, and hormone therapy, have been explored to reduce the rate of relapse, but have been unsuccessful. ${ }^{1}$ The proven efficacy of the anti-VEGFR tyrosine kinase inhibitors (TKIs) sunitinib and sorafenib in patients with mRCC supports their evaluation as adjuvant therapy, and multiple trials have evaluated this strategy. The ASSURE trial was the first large, multicentre, randomized, double-blind trial to report results of antiangiogenic therapies in the adjuvant setting for RCC. ${ }^{2}$ A total of 1943 patients who were considered to be at high risk for relapse following nephrectomy were randomly assigned to adjuvant treatment with sorafenib, sunitinib, or placebo. A high degree of Grade 3 or higher side effects and the need for dose reductions prompted a study amendment to reduce the starting dose to improve compliance. However, more than half of patients still experienced Grade 3 or higher side effects, and ASSURE failed to show improvements in the primary endpoint of disease-free survival (DFS) or in overall survival (OS) with either treatment vs. placebo.

In the S-TRAC trial, 615 patients with locoregional, highrisk RCC after nephrectomy were randomly assigned to treatment with sunitinib or placebo on a four-weeks on/twoweeks off schedule for one year or until disease recurrence, unacceptable toxicity, or consent withdrawal. ${ }^{3}$ Patients enrolled in this trial had more locoregionally advanced disease than that of the ASSURE trial. Patients treated with adjuvant sunitinib had a prolonged DFS compared with those given placebo (6.8 vs. 5.6 years; $p=0.03$ ); however, this was achieved at a cost of increased toxicities, including higher rates of Grade 3 or 4 adverse events (AEs) $(63.4 \%$ vs. $17.1 \%$ in placebo) and consistently lower health-related quality of life (HRQOL) scores compared with those who received placebo. Differences in outcomes between the ASSURE trial and the S-TRAC trial were likely related to differences in patient populations and methodologies. The ASSURE trial included many patients with stage 1 tumours $(9 \%)$ and patients with non-clear cell histology $(21 \%)$, while S-TRAC included only patients with late-stage (locoregional), clear-cell disease. In ASSURE, the starting dose of sunitinib was changed midtrial from $50 \mathrm{mg}$ to $37.5 \mathrm{mg}$, with dose 
reductions to $25 \mathrm{mg}$ allowed. In S-TRAC, the starting dose of $50 \mathrm{mg}$ was used, with dose reductions allowed to $37.5 \mathrm{mg}$ per day. S-TRAC also confirmed disease-free status before enrollment by central review of radiographs, with the primary endpoint of DFS based on blinded central review, while in ASSURE, assessments were conducted by investigators alone. Currently, OS in the S-TRAC trial is too immature to draw any conclusions.

A number of other trials are evaluating the use of antiVEGF TKIs and mTOR inhibitors in the adjuvant setting (Table 1). Their results are eagerly awaited and will hopefully provide guidance to the medical community about the most appropriate use of these agents in the adjuvant setting. It is likely that better means of patient selection will be needed to maximize the benefits of adjuvant treatments. Based on the clinical trial data so far, adjuvant use of sunitinib is likely to be limited to those with higher-risk disease (i.e., node-positive disease, larger T4 tumours, etc), especially if OS benefit is confirmed. Whether patients with lowerrisk disease (i.e., T2/T3 tumours) will benefit from adjuvant therapies remains to be determined and for most patients, surveillance remains the standard of care.

\section{Biomarkers}

For resected localized disease, use of a recurrence score, such as that proposed by Rini et al using a 16-gene assay, might also be incorporated into future clinical trials to further stratify patients with clear-cell RCC and to help identify patients with higher biological risk of recurrence. ${ }^{4}$ Eventually, this could help physicians make more informed treatment decisions about who is most appropriate for adjuvant therapies when they become available.

For metastatic disease, given the high cost of targeted therapies and the associated toxicities, identification of biomarkers to response may lead to improved identification of patients who are most likely (or least likely) to benefit from a particular treatment. For example, the mTOR inhibitors everolimus and temsirolimus have proven benefits in patients with mRCC. Recent data have suggested that mutations in the mTOR pathway genes MTOR, TSC1, and TSC2 are associated with a better response to rapalogues in patients with mRCC. ${ }^{5}$ The randomized, phase 2 RECORD-3 trial compared first-line everolimus followed by sunitinib with first-line sunitinib followed by everolimus at progression in 471 treatment-naïve patients with mRCC. At ASCO 2015, Hsieh and colleagues ${ }^{6}$ reported results of the RECORD-3 trial showing that mutations in $\mathrm{KDM} 5 \mathrm{C}$ may predict sensitivity to everolimus or sunitinib. KDM5C mutations were associated with longer progression-free survival (PFS) in those treated with sunitinib. ${ }^{6}$

Programmed death-ligand 1 (PD-L1) has also been explored as a biomarker to identify adverse clinicopathological features of RCC. ${ }^{7}$ PD-L1 expression in RCC is associated with aggressive features, such as higher TNM stage, tumour size or Fuhrman nuclear grade (FNG), and increased risk of cancer-specific mortality. However, the use of PD-L1 as a biomarker is constrained by a number of issues, as it may be affected by the age of specimens, the use of primary vs. metastatic tissue samples, and the notion that the immune system and the PD-1 axis may be a dynamic, ever-changing process that may not be captured well in a one-time test. ${ }^{8}$

None of these biomarkers on their own is able to positively select who will benefit from a particular drug because certainly there are patients without these mutations who still benefit from these drugs. To improve upon the limited predictive power of individual biomarker candidates, in the future, a panel of multiple markers may be required to generate more sensitive and more specific composite biomarker scores for predicting treatment responses and outcomes.

\section{Metastatic RCC}

There are several ongoing studies that may substantially alter the current approach to first-line management of mRCC. Two phase 3 trials are exploring anti-VEGFR TKI therapy in combination with cancer vaccines. IMA901, a vaccine comprised of tumour-associated peptides (TUMAPs), was

\begin{tabular}{|c|c|c|c|c|c|}
\hline Trial & $\mathbf{n}$ & Study design & $\begin{array}{l}\text { Primary } \\
\text { endpoint }\end{array}$ & $\begin{array}{l}\text { Year } \\
\text { started }\end{array}$ & Results \\
\hline ASSURE $^{2}$ & 1943 & $\begin{array}{l}\text { Placebo vs. sunitinib vs. } \\
\text { sorafenib, } 1 \text { year }\end{array}$ & DFS & 2006 & No difference in DFS or OS vs. placebo \\
\hline SORCE $^{15}$ & 1650 & $\begin{array}{l}\text { Placebo vs. sorafenib, } 1 \text { year, } \\
\text { vs. sorafenib, } 3 \text { years }\end{array}$ & DFS & 2007 & Results awaited \\
\hline S-TRAC ${ }^{3}$ & 500 & Placebo vs. sunitinib, 1 year & DFS & 2007 & $\begin{array}{l}\text { Prolonged DFS vs. placebo ( } 6.8 \text { vs. } 5.6 \text { years; } p=0.03 \text { ) } \\
\text { Higher rates of Grade } 3 \text { or } 4 \text { AEs }(63.4 \% \text { vs. } 17.1 \%) \\
\text { Lower HRQOL scores }\end{array}$ \\
\hline Everest $^{16}$ & 1218 & Placebo vs. everolimus, 1 year & DFS & Q3 2010 & Still recruiting \\
\hline PROTECT VEG113387 ${ }^{17}$ & 1500 & Placebo vs. pazopanib, 1 year & DFS & Q4 2010 & Results awaited \\
\hline ATLAS $^{18}$ & 700 & Placebo vs. axitinib, 3 years & DFS & Q2 2012 & Still recruiting \\
\hline
\end{tabular}

AE: adverse event; DFS: disease-free survival; HROOL: health-related quality of life; OS: overall survival. 
a negative study. ${ }^{9}$ Another vaccine, AGS-003, is currently in late-stage development for mRCC. The phase 3 ADAPT trial will compare sunitinib monotherapy with sunitinib in combination with AGS-003 in 450 patients with synchronous mRCC. ${ }^{10}$

In addition to vaccines, other immunotherapeutic approaches are being explored in the first-line treatment of mRCC. There is increasing attention on the PD-1 signaling pathway. The PD-1 inhibitor nivolumab, now a secondline standard of care for mRCC, was evaluated in the phase 3 registration CheckMate-025 trial comparing nivolumab monotherapy with everolimus in patients who had received one or two prior antiangiogenic therapies. ${ }^{11}$ There was an improvement in OS with nivolumab (25 months vs. 19.6 months; hazard ratio [HR] 0.73), as well as an increase in objective response rate (ORR) $(25 \%$ vs. $5 \%)$ vs. everolimus, with no difference in PFS (4.6 vs. 4.4 months; HR 0.88). ${ }^{12} \mathrm{~A}$ phase 3 trial is currently evaluating the combination of nivolumab and ipilimumab in a first-line setting vs. sunitinib with OS as the primary endpoint, ${ }^{13}$ based on an acceptable safety profile and promising response rate of this combination in a phase 1 trial. ${ }^{14}$ Numerous other combination trials with PD-1 inhibitors and VEGFR inhibitors are ongoing with agents including atezolizumab, avelumab, durvalumab, axitinib, cabozantinib, and lenvatinib. Their results are eagerly awaited; however, unless there is a very significant complete response rate or large OS advantage, the cost and toxicities associated with these combination therapies may preclude their widespread use.

\section{Conclusion}

The management of RCC has evolved markedly over the past decade, with the introduction of the targeted therapies sunitinib, sorafenib, pazopanib, and temsirolimus. The next 10 years hold promise for even greater expansion of the therapeutic armamentarium for RCC. With a number of recently completed and ongoing trials exploring anti-VEGF TKIs and mTOR inhibitors in the adjuvant setting, there is great potential for these agents to move from the setting of metastatic disease to the adjuvant space. With somewhat conflicting outcomes in the first large adjuvant trials of sunitinib and sorafenib, appropriate patient selection will become increasingly important and awaiting other adjuvant trials to report will help better inform our practice. Currently, robust predictive biomarkers used to guide personalized medicine are lacking. Given the high cost of the available and emerging drugs and the need to avoid unnecessary toxicities in patients who are unlikely to benefit, future studies will need to identify patients who are most likely to benefit. The coming years also hold promise with the emergence of new systemic treatments and combination therapies for mRCC, many of which show promise in the front-line setting.

\section{References}

1. Janowitz T, Welsh SJ, Zaki K, et al. Adjuvant therapy in renal cell carcinoma-past, present, and future. Semin Oncol 2013;40:482-91. htrps://doi.org/10.1053/i.seminoncol.2013.05.004

2. Haas NB, Manola J, Ky B, et al. Effects of adjuvant sorafenib and sunitinib on cardiac function in renal cell carcinoma patients without overt metastases: Results from ASSURE, ECOG 2805. Clin Cancer Res 2015;21:4048-54. https://doi.org/10.1158/1078-0432.CCR-15-0215

3. Ravaud A, Motzer RJ, Pandha HS, et al. Adjuvant sunitinib in high-risk renal cell carcinoma after nephrectomy. N Engl J Med 2016 (Epub before print).

4. Rini B, Goddard A, Knezevic D, et al. A 16-gene assay to predict recurrence ofter surgery in localized renal cell carcinoma: Development and validation studies. Lancet Oncol 2015;16:676-85. https://doi. org/10.1016/S1470-2045(15)70167-1

5. Kwiatkowski DJ, Choueiri TK, Fay AP, et al. Mutations in TSC1, ISC2, and MTOR are associated with response to rapalogs in patients with metastatic renal cell carcinoma. Clin Cancer Res 2016;22:2445-52. https://doi.org/10.1158/1078-0432.CCR-15-2631

6. Hsieh J, Chen D, Wang P, et al. Identification of efficacy biomarkers in a large metastatic renal cell carcinoma (mRCC) cohort through next generation sequencing (NGS): Results from RECORD-3. I Clin Oncol 2015;33(suppl): abstract 4509.

7. $X_{\cup} F, X \cup L$, Wang $Q$, et al. Clinicopathological and prognostic value of programmed death ligand-7 (PD-LI) in renal cell carcinoma: A meta-analysis. Int I Clin Exp Med 2015;8:14595-603.

8. Callea M, Abiges L, Gupta $M$, et al. Differential expression of PD-L1 between primary and metastatic sites in clear-cell renal cell carcinoma. Cancer Immunol Res 2015;3:1158-64. https://doi.org/10.1158/23266066.CIR-15-0043

9. NCT01265901. A randomized, controlled, phase 3 study investigating IMA901 multipeptide cancer vaccine in patients receiving sunitinib as first-line therapy for advanced/metastatic renal cell carcinoma. http://clinicaltrials.gov/ct2/show/NCT01265901? termZIMA-901\&rankZ2. Accessed November 17, 2016

10. Figlin RA, Nicolette CA, Amin A, et al. Monitoring T-cell responses in a phase 2 study of AGS-003, an autologous dendritic cell-based therapy in patients with newly diagnosed advanced stage renal cell carcinoma in combination with sunitinib. J Clin Oncol 201 1;29(suppl):abstract 2532

11. NCT01668784. Study of nivolumab (BMS-936558) vs. everolimus in pre-treated advanced or metastatic clear-cell renal cell carcinoma (CheckMate-025). https://clinicaltrials.gov/c+2/show/NCT01668784?† erm=CheckMate+025\&rank=1. Accessed November 17, 2016.

12. Motzer R, Sharma P, McDermott D, et al. CheckMate-025 phase 3 trial: Outcomes by key baseline factors and prior therapy for nivolumab (NIVO) vs. everolimus (EVE) in advanced renal cell carcinoma (RCC). J Clin Oncol 2016;34(Suppl 2S):abstract 498.

13. NCT02231749. Nivolumab combined with ipilimumab vs. sunitinib in previously untreated advanced or metastatic renal cell carcinoma (CheckMate-214). https://clinicaltrials.gov/ct2/show/NCT02231749. Accessed November 17, 2016.

14. Hammers $\mathrm{H}$, Plimack $\mathrm{E}$, Infante J, et al. Phase 1 study of nivolumab in combination with ipilimumab in metastatic renal cell carcinoma (mRCC). J Clin Oncol 2014;32(Suppl15):abstract 4504.

15. NCT00492258. Sorafenib in treating patients at risk of relapse after undergoing surgery to remove kidney cancer. https://clinicaltrials.gov/c+2/show/NCT00492258? term=sorce+sorafenib\&rank=1. Accessed November 17, 2016.

16. NCT01120249. Everolimus in treating patients with kidney cancer who have undergone surgery. https://clinicaltrials.gov/ct2/show/NCT01120249? term=NCT01 120249\&rank=1. Accessed November $17,2016$.

17. NCT01235962. A Study to evaluate pazopanib as an adjuvant treatment for localized renal cell carcinoma (RCC) (PROTECT). https://dinicaltrials.gov/ct2/show/NCT01235962? term=NCT012359628rank=1. Accessed November 17, 2016.

18. NCT01599754. Adjuvant axitinib therapy of renal cell cancer in high-risk patients (ATLAS). https://clinicaltrials.gov/c†2/show/NCT01599754?term=NCT01599754\&. Accessed November 17, 2016 